\title{
RATIONAL APPROXIMATION OF EXTREMAL LENGTH FOR DOUBLY CONNECTED DOMAINS
}

\author{
C. WAYNE MASTIN
}

\begin{abstract}
Results on the approximation of analytic functions are used to approximate the extremal length of the family of curves separating the boundary components of a doubly connected domain. Bounds for the approximations are established.
\end{abstract}

1. Introduction. In this report we will consider the problem of approximating the extremal length of the family of curves separating the boundary components of a doubly connected domain. A method of successive approximations will be developed using a basis for the Hilbert space of square-integrable analytic functions on the domain. It will be shown that the extremal length can be obtained from the solution of a minimization problem in this infinite-dimensional space and, furthermore, that this solution can be approximated by solving a minimization problem in a finite-dimensional subspace. In the problem for the whole space there are an infinite number of constraints, but in the problem for the subspace there are only a finite number of constraints.

The method of approximation which will be developed here is somewhat similar to that of the approximation of the solution of the minimum problem associated with the Bergman kernel function. That method may be found in the book by Kantorovich and Krylov [2].

2. The extremal length and module of a doubly connected domain. Let $D$ be a bounded doubly connected domain bounded by analytic curves and let $\Gamma$ be a family of simple closed contours in $D$ which separate the boundary components of $D$. The module of $\Gamma$ is defined to be

$$
m(\Gamma)=\inf \left\{\iint_{D} \rho^{2} \mid \rho \in U\right\}
$$

where $U$ is the set of all nonnegative measurable functions on $D$ such that, for each $\gamma$ in $\Gamma, \rho$ is a measurable function of arc length on $\gamma$ and the integral with respect to arc length $\int_{\gamma} \rho \geqq 1$. The extremal length of $\Gamma$ is $\lambda(\Gamma)=[m(\Gamma)]^{-1}$.

Received by the editors September 29, 1972 and, in revised form, February 15, 1973 and May 14, 1973.

AMS (MOS) subject classifications (1970). Primary 30A82, 31A15. 
Let $C_{0}$ be the family of all simple closed contours separating the boundary components of $D$. We will call $m\left(C_{0}\right)$ the module of $D$. If $F$ is a one-to-one conformal mapping of $D$ onto the circular ring $1<|z|<r$, then

$$
m\left(C_{0}\right)=\iint_{D} \rho^{2} \text { and } \int_{c} \rho \geqq 1
$$

for every $c$ in $C_{0}$ if and only if $\rho=\left|F^{\prime}\right| / 2 \pi|F|$, except possibly on a set of measure zero. We note that $m\left(C_{0}\right)=(1 / 2 \pi) \log r$. The proofs of the above-mentioned results and other properties of module and extremal length may be found in the book by Jenkins [1].

Let $L^{2}(D)$ be the Hilbert space of square-integrable analytic functions on $D$ with inner product

$$
(f, g)=\iint_{D} f \bar{g} .
$$

The module of $D$ can now be characterized as

$$
m\left(C_{0}\right)=\inf \left\{(f, f) \mid f \in L^{2}(D) \text { and } \int_{c}|f| \geqq 1 \text { for every } c \text { in } C_{0}\right\} .
$$

As has been seen, the function $F^{\prime} / 2 \pi F$ gives the desired minimum value. If $f$ and $g$ are two minimizing functions, then $|f|=|g|$ and hence $f=e^{i \theta} g$ for some real number $\theta$.

3. Approximating the module by families of curves with bounded length. Let $K$ be a positive constant greater than the length of either boundary component of $D$. For each such $K$ we define the family $C(K)$ consisting of the curves in $C_{0}$ with length less than or equal to $K$.

THEOREM 3.1. There is a constant $K_{0}>0$ such that $m(C(K))=m\left(C_{0}\right)$ for all $K \geqq K_{0}$.

Proof. Let $R$ be the circular ring $1<|z|<r$ which is conformally equivalent to the domain $D$. Let $\Gamma$ be the collection of circles given by $z=\rho e^{i \theta}$ where $1<\rho<r$. Since the module of $R$ is $(1 / 2 \pi) \log r, m(\Gamma) \leqq$ $(1 / 2 \pi) \log r$. It can be readily shown, as in Jenkins $[1$, p. 18], that $f(z)=2 \pi / z$ possesses the properties

$$
\iint_{R}|f|^{2}=\frac{1}{2 \pi} \log r \text { and } \int_{\gamma}|f| \geqq 1 \text { for } \gamma \in \Gamma .
$$

Now let $f$ be any function in $L^{2}(R)$ such that $\int_{\gamma}|f| \geqq 1$ for $\gamma \in \Gamma$. Then 
since $\int_{\gamma}|f|=\rho \int_{0}^{2 \pi}\left|f\left(\rho e^{i \theta}\right)\right| d \theta$, as in Jenkins [1, p. 18],

$$
\frac{1}{2 \pi} \int_{0}^{2 \pi} \int_{1}^{r}\left|f\left(\rho e^{i \theta}\right)\right| d \rho d \theta \geqq \frac{1}{2 \pi} \log r=\frac{1}{4 \pi^{2}} \int_{0}^{2 \pi} \int_{1}^{r} \frac{1}{\rho} d \rho d \theta,
$$

which implies

$$
\begin{aligned}
0 & \leqq \int_{0}^{2 \pi} \int_{1}^{r}\left(\left|f\left(\rho e^{i \theta}\right)\right|-\frac{1}{2 \pi \rho}\right)^{2} \rho d \rho d \theta \\
& \leqq \int_{0}^{2 \pi} \int_{1}^{r}\left|f\left(\rho e^{i \theta}\right)\right|^{2} \rho d \rho d \theta-\frac{1}{\pi} \int_{0}^{2 \pi} \int_{1}^{r}\left|f\left(\rho e^{i \theta}\right)\right| d \rho d \theta+\frac{1}{4 \pi^{2}} \int_{0}^{2 \pi} \int_{1}^{r} \frac{1}{\rho} d \rho d \theta \\
& \leqq \int_{0}^{2 \pi} \int_{1}^{r}\left|f\left(\rho e^{i \theta}\right)\right|^{2} \rho d \rho d \theta-\frac{1}{2 \pi} \log r .
\end{aligned}
$$

Thus

$$
\iint_{R}|f|^{2} \geqq \frac{1}{2 \pi} \log r \text { and } m(\Gamma)=\frac{1}{2 \pi} \log r .
$$

Let $F$ be a one-to-one conformal mapping of $R$ onto $D$. Let $\Lambda$ be the image of $\Gamma$ under $F$. Then $m(\Lambda)=m(\Gamma)$ which implies $m\left(C_{0}\right)$, the module of $D$, is equal to $m(\Lambda)$ since $m(\Gamma)$ is equal to the module of $R$. For any $\lambda$ in $\Lambda$ there is a $\gamma$ in $\Gamma$ such that $f(\gamma)=\lambda$. Hence the length of $\lambda$, which is $\int_{\gamma}\left|F^{\prime}\right|$, satisfies

$$
\int_{\gamma}\left|F^{\prime}\right| \leqq 2 \pi r M
$$

where $M=\max \left\{\left|F^{\prime}(z)\right| \mid z \in R\right\}$. Let $K_{0}=2 \pi r M$. Then $m(\Lambda) \leqq m(C(K)) \leqq$ $m\left(C_{0}\right)$ for any $K \geqq K_{0}$ and since $m(\Lambda)=m\left(C_{0}\right), m(C(K))=m\left(C_{0}\right)$ for $K \geqq K_{0}$.

In the proof of the above theorem, a value for $K_{0}$ is found which depends on the conformal mapping function. Assuming this function is not known, we do not know exactly how large $K_{0}$ must be. It is sufficient that $K_{0}$ be larger than the maximum length of the images of the circles in $\Gamma$. If the original domain $D$ were the circular ring $1<|z|<r$, then $m(C(K))=$ $m\left(C_{0}\right)$ for all $K$ under consideration; that is, $K>2 \pi r$.

4. Approximating the module for curves with bounded length by rational functions. In the remaining part of this paper, we assume that $K$ is large enough so that $m(C(K))=m\left(C_{0}\right)$. The family $C(K)$ will be denoted simply by $C$. This section deals with approximating $m(C)$ which is equal to the module of $D$.

Let $\left\{C_{n}\right\}$ be a sequence of finite families of curves such that

$$
C_{1} \subset C_{2} \subset \cdots \subset C
$$


and, for any given $c$ in $C, c: z=\varphi(t), 0 \leqq t \leqq 1$, and $\varepsilon>0$, there exists an $N>0$ so that whenever $n>N$ we can find a $c_{n}$ in $C_{n}, c_{n}: z=\varphi_{n}(t), 0 \leqq t \leqq 1$, with $\left|\varphi_{n}(t)-\varphi(t)\right|<\varepsilon$ for $0 \leqq t \leqq 1$ and $\left|\varphi_{n}^{\prime}(t)-\varphi^{\prime}(t)\right|<\varepsilon$ if $\varphi_{n}^{\prime}(t)$ and $\varphi^{\prime}(t)$ are both defined. The following is an example of such a sequence. For each positive integer $n$, let $P_{n}$ be a finite set of points in $D$ with $P_{1} \subset P_{2} \subset \cdots$ and $\bigcup_{n=1}^{\infty} P_{n}$ dense in $D$. If $C$ is the family of simple closed polygonal paths contained in $C$ with vertices in $P_{n}$, then $\left\{C_{n}\right\}$ satisfies the above conditions.

Let $\left\{w_{k}\right\}$ be a basis for $L^{2}(D)$ consisting of rational functions with all poles at a single point $z=\alpha$ in the bounded component of the complement of the closure of $D$ (see Meschkowski [3, p. 83]). We will be concerned with the relation between the following quantities and the module of $D$ :

$$
\begin{aligned}
& m\left(C_{n}\right)=\inf \left\{(f, f) \mid f \in L^{2}(D) \text { and } \int_{c}|f| \geqq 1 \text { for } c \text { in } C_{n}\right\}, \\
& m_{k}(C)=\inf \left\{(f, f) \mid f=\sum_{i=1}^{k} a_{i} w_{i} \text { and } \int_{c}|f| \geqq 1 \text { for } c \text { in } C\right\}, \\
& m_{k}\left(C_{n}\right)=\inf \left\{(f, f) \mid f=\sum_{i=1}^{k} a_{i} w_{i} \text { and } \int_{c}|f| \geqq 1 \text { for } c \text { in } C_{n}\right\}
\end{aligned}
$$

Each of the above is a positive finite quantity and in each case the minimum value is assumed. From the definitions we have $m\left(C_{n}\right) \leqq m(C) \leqq m_{k}(C)$, $m\left(C_{n}\right) \leqq m_{k}\left(C_{n}\right) \leqq m_{k}(C)$

THEOREM 4.1. $\lim _{k, \infty} m_{k}(C)=m(C)$.

Proof. We have already noted that $m(C) \leqq m_{k}(C)$ for any $k$. Suppose $m_{k}(C)$ does not converge to $m(C)$. Since $\left\{m_{k}(C)\right\}$ is a monotone sequence, there is an $\varepsilon>0$ such that $m_{k}(C)>m(C)+\varepsilon$ for every $k$.

Let $f$ be the minimizing function $F^{\prime} / 2 \pi F$ as given in $\S 2$. Then since $C \subset C_{0}$ and $m(C)=m\left(C_{0}\right)$, we have a function $f$ in $L^{2}(D)$ with $\int_{c}|f| \geqq 1$ for every $c$ in $C$ and $(f, f)=m(C)$. From the relation between $f$ and the conformal mapping function given in $\S 2$ and the fact that $D$ is bounded by analytic curves, we observe that $f$ is analytic on the closure of $D$. Thus $f$ can be expanded in a series $\sum_{i=1}^{\infty} a_{i} w_{i}$ which converges uniformly to $f$ on $D$. Let $f_{n}=\sum_{i=1}^{n} a_{i} w_{i}$. Let $\delta$ be a positive number less than $1 / K$ and $N_{\delta}$ be a positive integer such that $\left|f_{n}-f\right|<\delta$ whenever $n>N_{\delta}$. Let $A$ denote the area of $D$ and let $M$ be a bound for $\left\{f_{n}\right\}$ on $D$. Then

$$
\left|\left(f_{n}, f_{n}\right)-(f, f)\right| \leqq 2 M \iint_{D}\left|f_{n}-f\right|<2 M A \delta
$$


for $n>N_{\delta}$. Also

$$
1-\int_{c}\left|f_{n}\right| \leqq \int_{c}|f|-\int_{c}\left|f_{n}\right| \leqq \int_{c}\left|f-f_{n}\right| \leqq K \delta
$$

for $c$ in $C$ and $n>N_{\delta}$. Thus $\int_{c}\left|f_{n}\right| \geqq 1-K \delta>0$. Let $g_{n}=(1-K \delta)^{-1} f_{n}$. Now

$$
\int_{c}\left|g_{n}\right| \geqq 1
$$

and

$$
\begin{aligned}
\left(g_{n}, g_{n}\right)-\left(f_{n}, f_{n}\right) & =\left[(1-K \delta)^{-2}-1\right]\left(f_{n}, f_{n}\right) \\
& <\left[(1-K \delta)^{-2}-1\right][(f, f)+2 A M \delta]
\end{aligned}
$$

Hence, for $n>N_{\delta}$,

$$
\left|\left(g_{n}, g_{n}\right)-(f, f)\right| \leqq\left|\left(g_{n}, g_{n}\right)-\left(f_{n}, f_{n}\right)\right|+\left|\left(f_{n}, f_{n}\right)-(f, f)\right|<\eta
$$

where $\eta=2 A M \delta+\left[(1-K \delta)^{-2}-1\right][(f, f)+2 A M \delta]>0$. Choose $\delta$ small enough so that $\eta<\varepsilon / 2$. Then for $n>N_{\delta}$,

$$
m_{n}(C)>m(C)+\varepsilon=(f, f)+\varepsilon>\left(g_{n}, g_{n}\right)+\varepsilon / 2 .
$$

This contradicts the definition of $m_{n}(C)$.

THEOREM 4.2. $\lim _{n \rightarrow \infty} m\left(C_{n}\right)=m(C)$.

Proof. For each positive integer $n$ there is a function $f_{n}$ in $L^{2}(D)$ such that $\left(f_{n}, f_{n}\right)=m\left(C_{n}\right)$ and $\int_{c}\left|f_{n}\right| \geqq 1$ for every $c$ in $C_{n}$. Since $m\left(C_{n}\right) \leqq$ $m(C)$ for each $n$, the sequence $\left\{\left(f_{n}, f_{n}\right)\right\}$ is bounded. This implies that the sequence of functions $\left\{f_{n}\right\}$ is uniformly bounded on compact subsets of $D$ and hence is a normal family. Now there is a subsequence $\left\{f_{n_{p}}\right\}$ of $\left\{f_{n}\right\}$ which converges to a function $f$ in $L^{2}(D)$ uniformly on compact subsets of $D$. By considering an exhaustion of $D$ by compact subsets, it can be shown that

$$
\liminf _{p \rightarrow \infty}\left(f_{n_{\mathcal{D}}}, f_{n_{\mathcal{D}}}\right) \geqq(f, f) .
$$

Also, from the definition of $C_{n}$, it is evident that $\int_{c}|f| \geqq 1$ for every $c$ in $C$. Thus $(f, f) \geqq m(C)$ which implies

$$
\liminf _{\nu \rightarrow \infty} m\left(C_{n_{\nu}}\right) \geqq m(C) .
$$

Since $m\left(C_{n}\right) \leqq m(C)$ and $\left\{m\left(C_{n}\right)\right\}$ is a monotone sequence, we have

$$
\lim _{n \rightarrow \infty} m\left(C_{n}\right)=m(C) .
$$

THEOREM 4.3. $\lim _{n, k \rightarrow \infty} m_{k}\left(C_{n}\right)=m(C)$. 
Proof. By Theorems 4.1 and 4.2 ,

$$
m(C)=\lim _{k \rightarrow \infty} m_{k}(C)=\lim _{n \rightarrow \infty} m\left(C_{n}\right)
$$

The result now follows since $m\left(C_{n}\right) \leqq m_{k}\left(C_{n}\right) \leqq m_{k}(C)$.

5. Bounds for $m(C)$ and $m_{k}\left(C_{n}\right)$. An upper bound for $M(C)$ is first obtained involving the Bergman kernel function for the domain $D$. Let $\zeta$ be an arbitrary point in $D$ and let $f_{\zeta}$ be the unique function with minimum norm from among all functions in $L^{2}(D)$ assuming the value 1 at $z=\zeta$. This function is related to the Bergman kernel function $K(z, \zeta)$ by the equation

$$
f_{\zeta}(z)=K(z, \zeta) / K(\zeta, \zeta)
$$

Since $D$ is doubly connected, $K(z, \zeta)$ does not have a single-valued integral and hence $\int_{c} K(z, \zeta) d z \neq 0$ for $c \in C$. We also note that $K(\zeta, \zeta)>0$. The verification of the above results are found in the book by Meschkowski [3, Chapter 4].

Since, for all $c$ in $C$,

$$
\int_{c}\left|f_{\zeta}\right| \geqq\left|\int_{c} f_{\zeta}(z) d z\right|=\frac{1}{K(\zeta, \zeta)}\left|\int_{c} K(z, \zeta) d z\right|>0
$$

and the last quantity is constant for $c$ in $C$, we have

$$
I=\inf \left\{\int_{c}\left|f_{\zeta}\right| \mid c \in C\right\}>0 \text {. }
$$

Now $\int_{c} I^{-1}\left|f_{\zeta}\right| \geqq 1$, which implies $I^{-2} \iint_{I}\left|f_{\zeta}\right|^{2} \geqq m(C)$. If we let

then since $J \leqq I$ and

$$
J=\left|\int_{c} f_{\zeta}(z) d z\right|
$$

it follows that

$$
\iint_{D}\left|f_{\zeta}\right|^{2}=1 / K(\zeta, \zeta)
$$

$$
1 / J^{2} K(\zeta, \zeta) \geqq 1 / I^{2} K(\zeta, \zeta) \geqq m(C)
$$

Thus the module $m(C)$ of $D$ satisfies the inequality

$$
\frac{1}{m(C)} \geqq J^{2} K(\zeta, \zeta)=\frac{1}{K(\zeta, \zeta)}\left|\int_{c} K(z, \zeta) d z\right|^{2}
$$

where $c$ is an arbitrary curve in $C$.

A similar result for $m_{k}\left(C_{n}\right)$ can be obtained by using the reproducing kernel $K_{k}(z, \zeta)$ for the subspace of $L^{2}(D)$ generated by the basis elements 
$w_{1}, \cdots, w_{k}$. In this case we have

$$
\frac{1}{m_{k}\left(C_{n}\right)} \geqq \frac{1}{K_{k}(\zeta, \zeta)}\left|\int_{c} K_{k}(z, \zeta) d z\right|^{2}
$$

where $c$ is an arbitrary curve in $C$.

Since it is known that $K(z, \zeta)$ and $K_{k}(z, \zeta)$ are analytic functions of $z$ in the closure of $D$ whenever $\zeta$ is fixed in $D$, in each of the two inequalities we can also let $c$ be either of the boundary components of $D$.

In order to obtain a lower bound for $m_{k}\left(C_{n}\right)$, it will be assumed that the basis $\left\{w_{k}\right\}$ for $L^{2}(D)$ has been orthonormalized. Then

implies

$$
f=\sum_{i=1}^{k} a_{i} w_{i}
$$

Also

$$
(f, f)=\sum_{i=1}^{k}\left|a_{i}\right|^{2}
$$

$$
\int_{c}|f| \leqq \sum_{i=1}^{k}\left|a_{i}\right| \int_{c}\left|w_{i}\right| \quad \text { for } c \in C_{n} .
$$

Let $c_{j}, 1 \leqq j \leqq p_{n}$, denote the curves in $C_{n}$. If we put $A_{i}=\left|a_{i}\right|$ and $W_{i j}=\int_{c}\left|w_{i}\right|$, then there is a unique $k$-tuple $\left(A_{1}, \cdots, A_{k}\right)$ which minimizes $\sum_{i=1}^{k} A_{i}^{2}$ from among all $k$-tuples with $\sum_{i=1}^{k} A_{i} W_{i j} \geqq 1,1 \leqq j \leqq p_{n}$. Since $\int_{c_{j}}|f| \geqq 1$ implies $\sum_{i=1}^{k} A_{i} W_{i j} \geqq 1$, it follows that if $\left(A_{1}, \cdots, A_{k}\right)$ is a solution to this minimum problem, then

$$
m_{k}\left(C_{n}\right) \geqq \sum_{i=1}^{k} A_{i}^{2} .
$$

We have thus obtained upper and lower bounds for $m_{k}\left(C_{n}\right)$. In the first case the bound involves a reproducing kernel which can be obtained by solving a minimum problem with a unique solution. In the second case we again solve a different minimum problem with a unique solution.

\section{BIBLIOGRAPHY}

1. J. A. Jenkins, Univalent functions and conformal mappings, Ergebnisse der Math. und ihrer Grenzgebiete, Heft 18, Springer-Verlag, Berlin, 1958. MR 20 \#3288.

2. L. V. Kantorovič and V. I. Krylov, Approximate methods of higher analysis, 3rd ed., GITTL, Moscow, 1950; English transl., Interscience, New York; Noordhoff, Groningen, 1958. MR 13, 77; 21 \#5268.

3. H. Meschkowski, Hilbertsche Räume mit Kernfunktion, Die Grundlehren der math. Wissenschaften, Band 113, Springer-Verlag, Berlin, 1962. MR 25 \#4326.

Department of Mathematics, Mississippi State University, Mississippi State, MISSISSIPPI 39762 